\title{
Effects of endometrial stem cell transplantation combined with estrogen in the repair of endometrial injury
}

\author{
XINRONG WANG $^{1 *}$, HONGCHU BAO $^{1 *}$, XUEMEI LIU ${ }^{1}, \mathrm{CHENGDE} \mathrm{WANG}^{2}$ and CUIFANG HAO ${ }^{1}$ \\ Departments of ${ }^{1}$ Reproduction Medicine and ${ }^{2}$ Thoracic Surgery, \\ The Affiliated Yantai Yuhuangding Hospital of Qingdao University, Yantai, Shandong 264000, P.R. China
}

Received August 15, 2017; Accepted February 27, 2018

DOI: $10.3892 / \mathrm{ol} .2018 .8702$

\begin{abstract}
The present study investigated the effects of endometrial stem cell (EnSCs) transplantation combined with estrogen in the repair of endometrial injury. A total of 30 patients with intrauterine adhesions (IUA) and 30 healthy individuals were selected. Expression of epidermal growth factor (EGF) and platelet-derived growth factor (PDGF)-BB in endometrial tissue was assessed. Additionally, expression levels of epithelial membrane antigen (EMA), cytokeratin (CK), integrin $\alpha-6$ (CD49f), Thy-1 membrane glycoprotein (THY-1), collagen type 1 (Col I), fibroblast (5B5) and vimentin in EnSCs were detected using western blot analysis and reverse transcription-quantitative polymerase chain reaction. A rat model of IUA was established and female rats were divided into the control, model, EnSCs, estrogen and estrogen plus EnSCs (E+EnSCs) groups. Blood was extracted at 1 and 5 weeks post-treatment, and serum levels of transforming growth factor (TGF)- $\beta 1, \mathrm{EGF}, 17 \beta$-estradiol (E2) and PDGF-BB were measured using ELISA. Hematoxylin and eosin staining was performed to observe the pathological changes of endometrial tissue in rats. Western blot analysis was used to detect the expression of estrogen receptor (ESR1) and matrix metalloproteinase (MMP)-9 in the endometrium. The results revealed that patients with IUA exhibited increased expression levels of EGF and PDGF-BB compared with those in control group. Additionally, EnSCs exhibited significantly increased expression levels of EMA, CD49f, CK, Col I, THY-1, $5 \mathrm{~B} 5$ and vimentin compared with the remaining groups. An increased number of newly formed glands was observed in the E+EnSCs group compared with that in the EnSCs group.
\end{abstract}

Correspondence to: Dr Cuifang Hao, Department of Reproduction Medicine, The Affiliated Yantai Yuhuangding Hospital of Qingdao University, 20 Yuhuangding East Road, Yantai, Shandong 264000, P.R. China

E-mail: haocuifang20178@126.com

${ }^{*}$ Contributed equally

Key words: intrauterine adhesions, endometrial stem cells, platelet-derived growth factor BB, estrogen, epidermal growth factor
Increased levels of E2, but decreased levels of TGF- $\beta 1$, EGF, PDGF-BB, ESR1 and MMP-9 were detected in EnSCs and estrogen groups compared with those in E+EnSCs group. These results suggest that EnSCs transplantation combined with estrogen could improve endometrial abnormalities.

\section{Introduction}

Intrauterine adhesions (IUA) refer to the endometrial fibrosis caused by endometrial injury in operative hysteroscopy (1). Endometrial injuries may lead to recurrent miscarriages, infertility, amenorrhea, abnormal placentation, dysmenorrhea and abnormal uterine bleeding, thus being detrimental to the health of affected women (2). Expression levels of transforming growth factor (TGF)- $\beta 1$, epidermal growth factor (EGF), basic fibroblast growth factor and platelet-derived growth factor (PDGF)-BB were found to be significantly increased in exudates in patients with IUA following surgery $(3,4)$, which in turn promoted the growth of fibroblasts and the formation of adhesions. Despite advances in intraoperative techniques, including minimizing trauma of the healthy myometrium and endometrium, and reducing the usage of electrosurgery (5), the incidence of IUA caused by surgery remains high. Therefore, the development of novel approaches to inhibit the formation of post-surgical IUA is required.

A variety of novel therapies for IUA have been developed. Estrogen is the most important female sex hormone, which serves pivotal functions in the regulation and development of the reproductive system of females and the formation of secondary sex characteristics. It has been reported that postoperative estrogen therapy may be used to prevent recurrent adhesions (6). However, the recurrence rate of IUA accounted for $62.5 \%$ in patients with severe IUAs (7). Transplantation of bone marrow mesenchymal stem cells (MSCs) may promote the regeneration of the injured uterus and abnormal endometrium (8-10). However, the application of MSCs in the treatment of IUA is limited due to its low efficiency in promoting endometrial repair and regeneration. It has been reported that human endometrial stem cells (EnSCs) derived from the endometrium may contribute to the repair of the injured endometrium by inducing angiogenesis (11), highlighting the potential of using ESCs for the treatment of IUA.

In the present study, a rat model of IUA was established. Additionally, the effects of hESCs, estrogen and the 
combination of estrogen plus hESCs in the treatment of IUA were assessed.

\section{Materials and methods}

Patients. A total of 30 patients aged 25-35 years (mean age: 31 ) with moderate to severe IUA underwent IUA dissection in the Affiliated Yantai Yuhuangding Hospital of Qingdao University (Shandong, China) between January 2016 and December 2016 and were enrolled in the present study. The criteria for patient inclusion were as follows: Initial hysteroscopic adhesions score in line with moderate to severe IUA [American Fertility Society score $(12) \geq 5$ ]; age $\leq 40$ years; hysteroscopic examination following surgery; and a complete record of the medical history. A total of 30 females aged 23-34 years (mean age: 28 ) with normal endometrium who received hysteroscopic examination were selected as controls. Endometrial tissue was collected by curettage. All participants provided written informed consent and the study was approved by the Ethics Committee of The Affiliated Yantai Yuhuangding Hospital of Qingdao University.

Reverse transcription-quantitative polymerase chain reaction (RT-qPCR). Expression levels of EGF and PDGF-BB in endometrial tissue were detected using RT-qPCR. Additionally, expression levels of epithelial membrane antigen (EMA), Thy-1 membrane glycoprotein (THY-1), cytokeratin (CK), collagen type 1 (Col I), integrin $\alpha-6$ (CD49f), vimentin and 5 B5 in ESCs were assessed using RT-qPCR. Total RNA was extracted from endometrial tissue and ESCs using TRIzol reagent (Thermo Fisher Scientific, Inc., Waltham, MA, USA). The concentration and quality of RNA were assessed using the 260/280 $\mathrm{nm}$ absorbance ratio of 1.8-2.0. Total RNA was reverse transcribed using a reverse transcription kit (Takara Bio, Inc., DRR047A) according to the manufacturer's protocol. RT-qPCR was performed using LightCycler ${ }^{\circledR} 480$ SYBR Green Master mix (Roche Diagnostics, Basel, Switzerland). Primers used for the PCR are listed in Table I. All primers were synthesized by Sangon Biotech Co., Ltd. (Shanghai, China). GAPDH was used as an endogenous control. Thermocycling conditions were as follows: $95^{\circ} \mathrm{C}$ for $30 \mathrm{sec}$, followed by 40 cycles of $95^{\circ} \mathrm{C}$ for $10 \mathrm{sec}, 60^{\circ} \mathrm{C}$ for $15 \mathrm{sec}$ and $72^{\circ} \mathrm{C}$ for $15 \mathrm{sec}$, and finally, $60^{\circ} \mathrm{C}$ for $15 \mathrm{sec}$. Relative expression levels were determined using the $2^{-\Delta \Delta \mathrm{Cq}}$ method (13).

Western blot analysis. Total protein was extracted from the endometrial tissues and EnSCs, and quantified using a bicinchoninic acid assay (Pierce; Thermo Fisher Scientific, Inc.). A total of $30 \mu \mathrm{g}$ protein/lane was separated by SDS-PAGE (10\% gels) and transferred onto polyvinylidene difluoride membranes. The membranes were then blocked with $5 \%$ non-fat milk. for $1 \mathrm{~h}$ at room temperature. Following blocking, the membranes were incubated with the following primary antibodies: Rabbit anti-human EGF antibody (cat. no. DF2225, 1:2,000; Affinity Biosciences, Cincinnati, OH, USA), rabbit anti-human PDGF-BB (cat. no. orb303833, 1:2,000; Biorbyt, Cambridge, UK,), rabbit anti-human EMA antibody (cat. no. orb31710; 1:2,000; Biorbyt), rabbit anti-human CK antibody (cat. no. BF0141, 1:1,000; Affinity Biosciences), rabbit anti-human CD49f antibody (cat. no. orb39949, 1:1,000;
Biorbyt), rabbit anti-human THY-1 (cat. no. orb136416, 1:2,000; Biorbyt), rabbit anti-human Col I antibody (cat. no. orb345868, 1:2,000; Biorbyt), rabbit anti-human 5B5 antibody (cat. no. orb241612, 1:1,000; Biorbyt), rabbit anti-human vimentin antibody (cat. no. AF7013, 1:2,000; Affinity Biosciences), rabbit anti-rat estrogen receptor (ESR1) (cat. no. DF6094, 1:2,000; Affinity Biosciences) and rabbit anti-rat matrix metalloproteinase (MPP)-9 (cat. no. orb100446, 1:1,000; Biorbyt) overnight at $4^{\circ} \mathrm{C}$. Membranes were washed three times with Tris-buffered-saline with Tween-20 (TBST) for $10 \mathrm{~min}$. Following the primary incubation, membranes were incubated with horseradish peroxidase-labeled goat anti-rabbit antibody (cat. no. orb345943, 1:1,500; Biorbyt) at room temperature for $1 \mathrm{~h}$. Membranes were then washed three times with TBST. Protein bands were visualized using enhanced chemiluminescence (ECL; Merck KGaA). The densitometric analysis for the quantification of the bands was performed using ImageJ software (version 2.1; National Institutes of Health, Bethesda, MD, USA). $\beta$-actin was used as an endogenous control.

Isolation and culture of human EnSCs. Endometrial tissue was obtained from ovulating women without endometrial disease. All participants provided written informed consent and the study was approved by the Ethics Committee of The Affiliated Yantai Yuhuangding Hospital of Qingdao University. EnSCs were prepared as reported by Ebrahimi-Barough et al $(14,15)$. Endometrial tissue was rinsed with PBS and cut into $1 \mathrm{~mm}^{3}$ pieces. Tissues were then incubated with $5 \mathrm{ml}$ digestion solution in a $25 \mathrm{~cm}^{2}$ cell culture flask [0.25\% trypsin (cat. no. T8150; Beijing Solarbio Science \& Technology Co, Ltd., Beijing, China) $+0.02 \%$ ethylenediaminetetraacetic acid (cat. no. C1033; Beijing Solarbio Science \& Technology Co., Ltd.) $+300 \mu \mathrm{g} / \mathrm{ml}$ type II collagenase (cat. no. C8150; Beijing Solarbio Science and Technology Co., Ltd.)] under agitation (80 rpm) for $45-60 \mathrm{~min}$ at $37^{\circ} \mathrm{C}$. Samples were filtered with 80 mesh screen and collect the filtrate. Red blood cells were removed with red blood cell lysis buffer (cat. no. ab204733; Abcam). When the cells were $\sim 80 \%$ confluent, they were used for subsequent experiments.

Immunocytochemistry. EnSCs were fixed with 4\% paraformaldehyde at room temperature for $20 \mathrm{~min}$ and permeabilised with $0.1 \%$ Triton in PBS at room temperature for $20 \mathrm{~min}$. The slides were then blocked using 5\% goat serum in TBS and incubated with primary antibody against CD90 (cat. no. ab106934, 1:200; Abcam) overnight at $4^{\circ} \mathrm{C}$. Following the primary incubation, cells were incubated with horseradish peroxidase secondary antibody (cat. no. ab95376; rabbit anti-rat immunoglobulin; 1:200; Abcam) at $37^{\circ} \mathrm{C}$ for $4 \mathrm{~h}$. Cells were washed with TBST for $10 \mathrm{~min}$. The negative controls were incubated only with the secondary antibody. The slides were stained with 3,3-diaminobenzidine tetrahydrochloride for $10 \mathrm{~min}$, counterstained with hematoxylin, subjected to gradient alcohol dehydration and mounted with neutral gum. Cells were observed under an optical microscope (x400 magnification).

Model establishment and treatment. A total of 50 specific pathogen-free (SPF) female Sprague Dawley rats (weight, 200-220 g, 8 weeks old) were purchased from the Shanghai 
Table I. Primers used in the study.

\begin{tabular}{|c|c|c|}
\hline Gene name & Primers & Sequence $\left(5^{\prime}-3^{\prime}\right)$ \\
\hline \multirow[t]{2}{*}{ EGF } & Sense & CAGGGAAGATGACCACCACT \\
\hline & Antisense & CAGTTCCCACCACTTCAGGT \\
\hline \multirow[t]{2}{*}{ PDGF-BB } & Sense & CGCGGATCCACCATGAATCGCTGCTGG \\
\hline & Antisense & CCGCTCGAGCTAGGCTCCAAGGGTCTC \\
\hline \multirow[t]{2}{*}{ EMA } & Sense & TGGATGTGCTTGATAAGCGG \\
\hline & Antisense & ACCATGTCCTTTCCAGTGTGT \\
\hline \multirow[t]{2}{*}{ CK } & Sense & GGTCATGGCCGAGCAGAA \\
\hline & Antisense & TTCAGTCCGGCTGGTGAAC \\
\hline \multirow[t]{2}{*}{ CD49f } & Sense & CCTGCTGCTGCTCCTCACA \\
\hline & Antisense & GTAACAACTGTTGCGGGTTTAGG \\
\hline \multirow[t]{2}{*}{ THY-1 } & Sense & ATCGCTCTCCTGCTAACAGTC \\
\hline & Antisense & CTCGTACTGGATGGGTGAACT \\
\hline \multirow[t]{2}{*}{ Col I } & Sense & AATCCTCTCGTCAAAACTGAAGG \\
\hline & Antisense & CCATCTCGCTTATCCAACAATGA \\
\hline \multirow[t]{2}{*}{$5 \mathrm{~B} 5$} & Sense & TGACAGCGACAAGAAGTG \\
\hline & Antisense & CAGTGAAGCGGTACATAGG \\
\hline \multirow[t]{2}{*}{ Vimentin } & Sense & ССАААСТTТТССТСССТGААСС \\
\hline & Antisense & GTGATGCTGAGAAGTTTCGTTGA \\
\hline \multirow[t]{2}{*}{ GAPDH } & Sense & GGCTCTCCAGAACATCATCC \\
\hline & Antisense & TGTCATCATATTTGGCAGGT \\
\hline
\end{tabular}

EGF, epidermal growth factor; PDGF-BB, platelet-derived growth factor-BB; TGF- $\beta 1$, transforming growth factor- $\beta 1$; ESR1, estrogen receptor; MMP-9, matrix metalloproteinase-9; EMA, epithelial membrane antigen; CK, cytokeratin; Col I, collagen type 1; THY-1, Thy-1 membrane glycoprotein; CD49F, integrin $\alpha-6$.

Institutes for Biological Sciences, Chinese Academy of Science (Shanghai, China). The animals were maintained in a 12-12 h light-dark cycle at a temperature of $22 \pm 1^{\circ} \mathrm{C}$ with free access to food and water in a specific pathogen-free environment. A total of 50 rats were randomly divided into 5 groups with 10 rats in each group. A rat model of IUA was established by scraping the uterine horn to mimic the cause of IUA in humans. The model was established using the methods described by Tang et al (16). Rats in the control group were subjected to a sham surgery. At day 7 following the establishment of the model, rats in the EnSCs group were subcutaneously injected with $1 \mathrm{ml}$ EnSCs, rats in estrogen group were subjected to subcutaneous injection of estradiol benzoate $(0.5 \mathrm{mg} / \mathrm{kg}$; Hangzhou Animal Pharmaceutical Factory, China) in PBS according to previous studies (17) and rats in the E+EnSCs group received an injection of estradiol benzoate $(0.5 \mathrm{mg} / \mathrm{kg})$ and EnSCs $(1 \mathrm{ml})$. Rats ( $\mathrm{n}=5 /$ group) were sacrificed 1 week after IUA and 5 weeks following IUA. Uterine tissue was collected from both sides of the uterus and $2 \mathrm{ml}$ of blood was extracted. Blood samples were centrifuged at 3,000 x g at $4^{\circ} \mathrm{C}$ for $10 \mathrm{~min}$. Serum was stored at $-80^{\circ} \mathrm{C}$. The study was approved by the Animal Ethics Committee of The Affiliated Yantai Yuhuangding Hospital of Qingdao University.

Hematoxylin and eosin staining. Uterine tissue was fixed in $10 \%$ neutral formaldehyde and embedded in paraffin. Paraffin-embedded tissue samples were cut into 5- $\mu \mathrm{m}$ sections.
The tissue sections were deparaffinized in xylene at room tempetature and rehydrated in a descending ethanol series (100\% for $5 \mathrm{~min}, 95 \%$ for $1 \mathrm{~min}, 80 \%$ for $5 \mathrm{~min}$ and $75 \%$ for $5 \mathrm{~min}$ ). Hematoxylin and eosin staining was performed using the routine method (18). Following dehydration, sections were treated with xylene for 2 times. Tissue sections were mounted with neutral resin and observed under optical microscopy (x200 magnification) (Olympus BX51; Olympus Corporation, Tokyo, Japan).

ELISA. The total protein extracted from the blood samples of the experimental rat groups was used to determin the serum concentrations of $\beta$-estradiol (E2) (cat. no. KGE014), TGF- $\beta 1$ (cat. no. MB100B), EGF (cat. no. DY3214) and PDGF-BB (cat. no. MBB00), as detected using ELISA kits (R\&D Systems, Inc., Minneapolis, MN, USA), according the manufacturer's protocol.

Statistical analysis. Data were analyzed using SPSS software (version 19.0; IBM Corp., Armonk, NY, USA). The relevant data are expressed as the mean \pm standard deviation. Results were analyzed using Student's t-test when only two groups were compared. Multiple comparisons between more than two groups were performed by one way analysis of variance followed by least significant difference or Kruskal-Wallis tests. $\mathrm{P}<0.05$ was considered to indicate a statistically significant difference. 
A

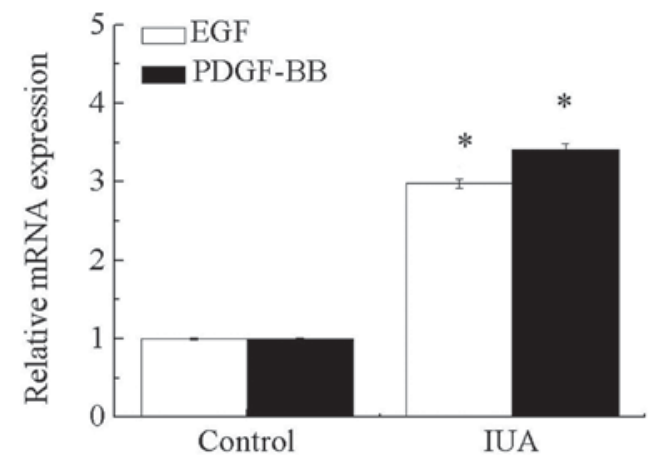

B

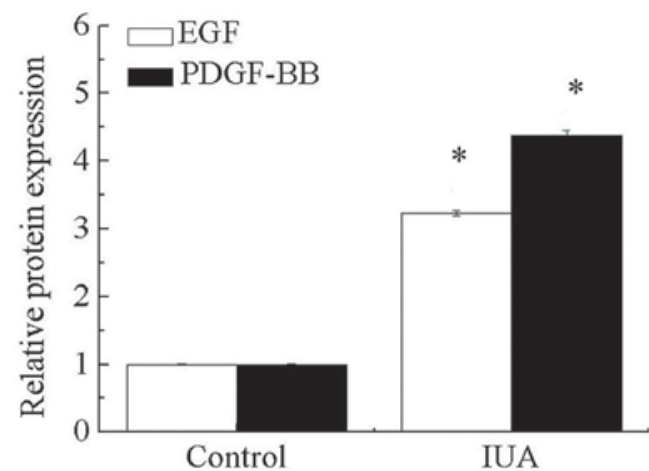

Figure 1. The mRNA and protein expression levels of EGF and PDGF-BB in endometrial tissue. (A) mRNA levels of EGF and PDGF-BB in endometrial tissue. (B) Protein expression levels of EGF and PDGF-BB in endometrial tissue. ${ }^{*} \mathrm{P}<0.05$ vs. control. EGF, epidermal growth factor; PDGF-BB, platelet-derived growth factor-BB; IUA, intrauterine adhesions.

\section{Results}

$m R N A$ and protein expression levels of EGF and PDGF-BB in IUA tissues. As presented in Fig. 1A and B, mRNA and protein levels of EGF and PDGF-BB were significantly increased in endometrial tissue derived from patients with IUA compared with that in healthy individuals $(\mathrm{P}<0.05)$. These results suggest that endometrial injury may significantly increase the expression levels of EGF and PDGF-BB.

Expression levels of EMA, CK, CD49f, THY-1, Col I, 5B5 and vimentin in EnSCs. As presented in Fig. 2, expression levels of endometrial epithelial stem cell-associated genes, including CK, EMA and CD49f, and endometrial stromal cell-associated genes, including THY-1 (CD90), Col I, 5B5 and vimentin, were significantly increased in EnSCs compared with that in the controls $(\mathrm{P}<0.05)$.

Characterization of isolated EnSCs. Isolated EnSCs were positive for CD90, as assessed using immunocytochemistry (Fig. 3A and B).

Morphological changes of the endometrium detected by hematoxylin and eosin staining. Hematoxylin and eosin staining demonstrated that the uterine cavity of the control group was covered by columnar epithelium and oval glands. In the model group, the uterine cavity was covered with flat columnar epithelial cells or stratified flat epithelial cells, and glands were rare. The number of endometrial glands in the estrogen, EnSCs and E+EnSCs groups was increased, and the newly formed glands were round or oval. No significant difference in the number of glands was detected between the EnSCs and estrogen groups. Newly formed glands were detected in the E+EnSCs group (as indicated by the black arrows; Fig. 4). These results suggest that treatment efficacy in the E+hESCs group may be improved compared with that in the remaining groups.

Expression levels of PDGF-BB, E2, EGF and TGF- $\beta 1$ in a rat model of IUA. As presented in Fig. 5A, no significant difference in the expression of E2 was detected 1 week after IUA. The results demonstrated that the levels of serum E2 in the estrogen, EnSCs and E+EnSCs groups were significantly higher than that in the control group $(\mathrm{P}<0.05)$. However, no significant differences were detected between the model and control groups. Additionally, the expression of E2 was significantly increased in the estrogen and E+EnSCs groups compared with that in the model group $(\mathrm{P}<0.05)$. Additionally, a significant decrease in the E2 content was detected in the EnSCs and estrogen groups compared with that in the $\mathrm{E}+\mathrm{EnSCs}$ group $(\mathrm{P}<0.05$; Fig. 5A).

One week after IUA, the levels of EGF, TGF- $\beta 1$ and PDGF-BB were significantly increased in the model, EnSCs, estrogen and $\mathrm{E}+\mathrm{EnSCs}$ groups compared with those in the control group (P<0.05; Fig. 5B-D). The levels of TGF- $\beta 1$, EGF and PDGF-BB were significantly decreased in the EnSCs, estrogen and $\mathrm{E}+\mathrm{EnSCs}$ groups compared with those in the model group at 4 weeks post-treatment $(\mathrm{P}<0.05$; Fig. 5B-D). No significant difference in the expression of EGF, TGF- $\beta 1$ and PDGF-BB was detected between the EnSCs and estrogen groups (Fig. 5B-D). Additionally, a significant decrease in the levels of TGF- $\beta 1$, EGF and PDGF-BB was detected in the E+EnSCs group compared with that in the EnSCs and estrogen groups ( $\mathrm{P}<0.05$; Fig. 5B-D). 

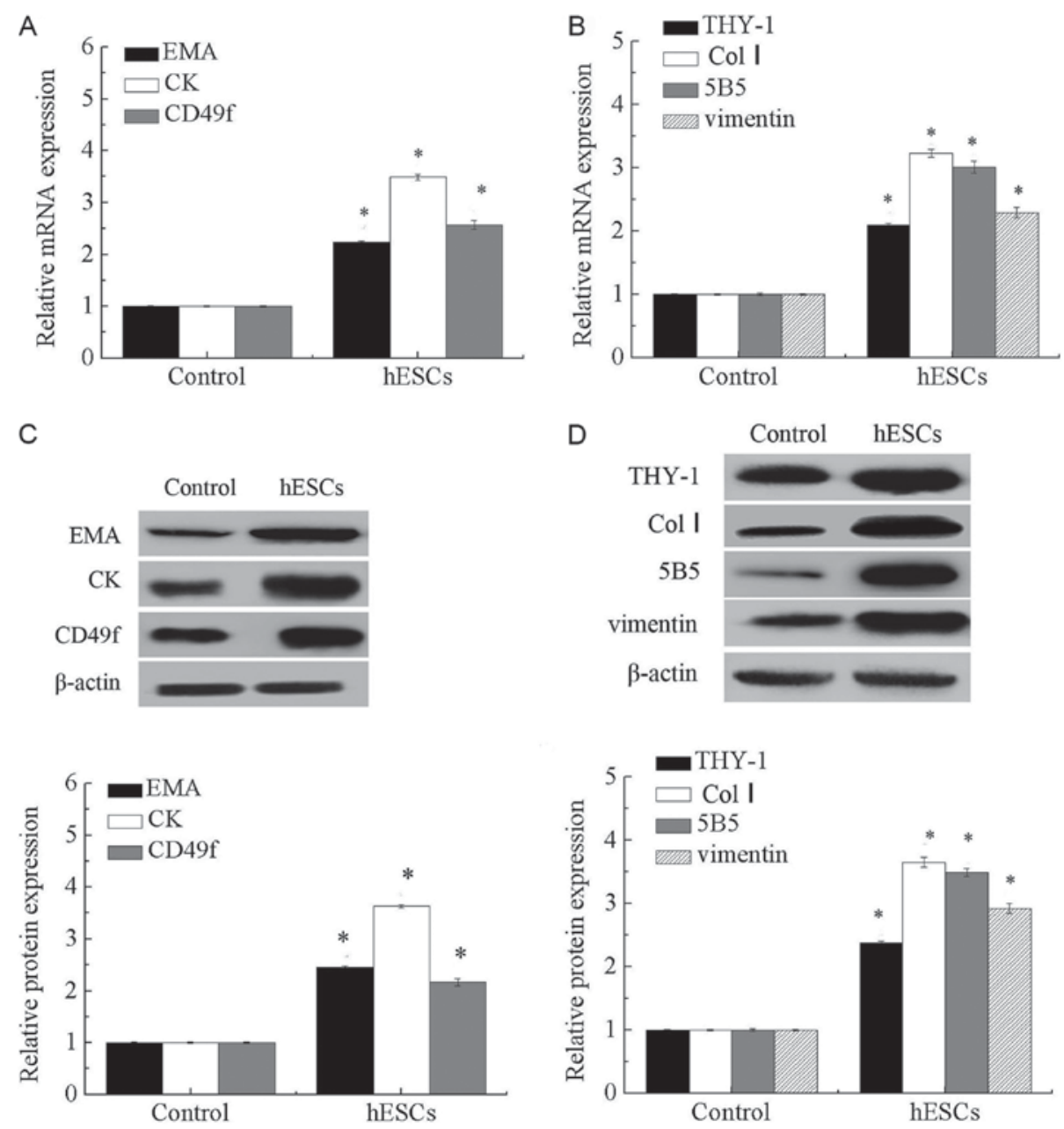

Figure 2. Expression of EMA, CK, CD49f, THY-1, Col I. 5B5 and vimentin in EnSCs. (A) mRNA levels of EMA, CK and CD49f in EnSCs. (B) mRNA levels of THY-1, Col I, 5B5 and vimentin in EnSCs. (C) Protein expression of EMA, CK and CD49f in EnSCs. (D) Protein expression of THY-1, Col I, 5B5 and vimentin in EnSCs. "P<0.05 vs. control. EMA, epithelial membrane antigen; CK, cytokeratin; Col I, collagen type 1; CD49F, integrin $\alpha-6$; THY-1, Thy-1 membrane glycoprotein; EnSCs, endometrial stem cells.
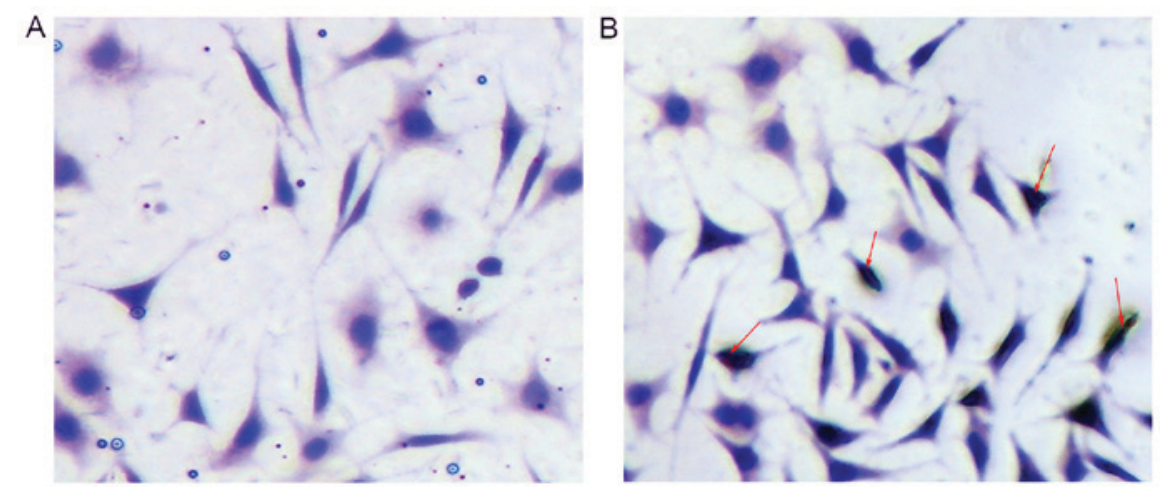

Figure 3. Immunocytochemical staining of endometrial stem cells. (A) Negative control group. (B) Immunostaining against cluster of differentiation 90 . Red arrows indicate positive cells. Magnification, x400.

Expression of ESRI and MMP-9 in endometrium in a rat model of IUA. One week after IUA, the expression levels of ESR1 and MMP-9 were significantly decreased in the model, EnSCs, estrogen and $\mathrm{E}+\mathrm{EnSCs}$ groups compared with those in the control group $(\mathrm{P}<0.05$; Fig. 6A-C), as assessed using western blot analysis. The expression levels of ESR1 and MMP-9 were significantly increased in the estrogen, EnSCs and E+EnSCs groups compared with those in model group at 5 weeks after IUA ( $\mathrm{P}<0.05$; Fig. 6B and $\mathrm{C})$. No significant difference in expression levels of ESR1 and MMP-9 were detected between the estrogen and EnSCs groups at 5 weeks after IUA. However, the expression levels of ESR1 and MMP-9 were significantly decreased in the estrogen and EnSCs groups compared with those in the E+EnSCs group ( $\mathrm{P}<0.05$; Fig. 6B and $\mathrm{C})$. 

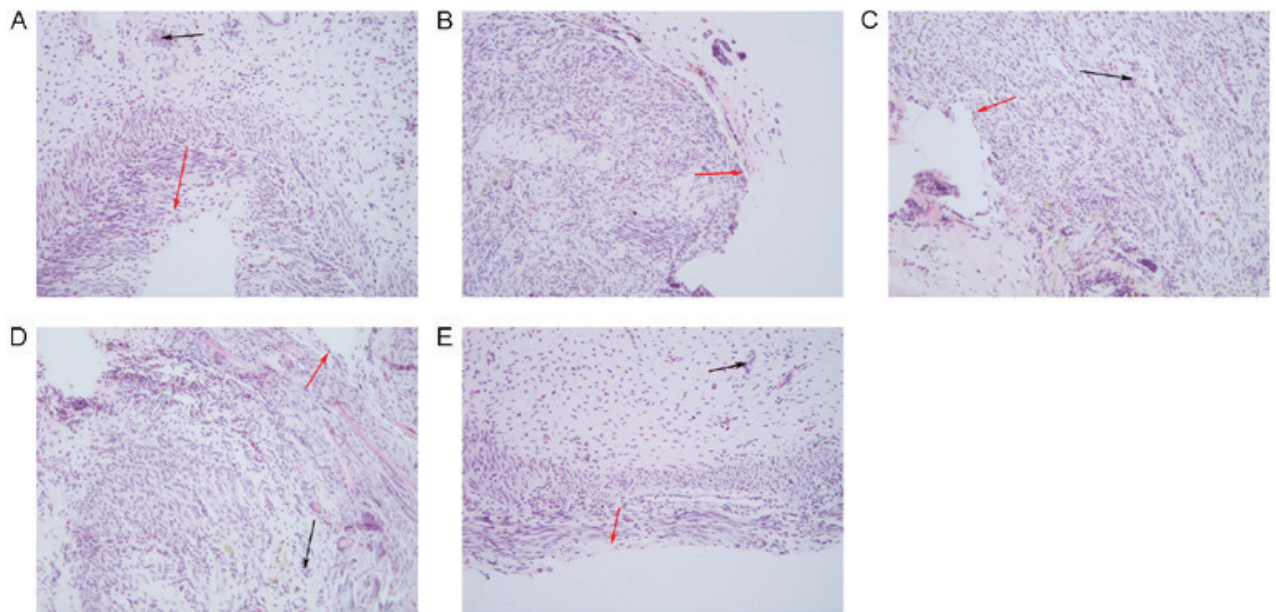

Figure 4. Morphological changes of endometrium detected by hematoxylin and eosin staining in (A) control, (B) model, (C) EnSCs, (D) estrogen and (E) E+EnSCS groups. Black arrows indicate glands, red arrows indicate epithelial cells. Magnification, x200. E+EnSCs, estrogen plus endometrial stem cells.
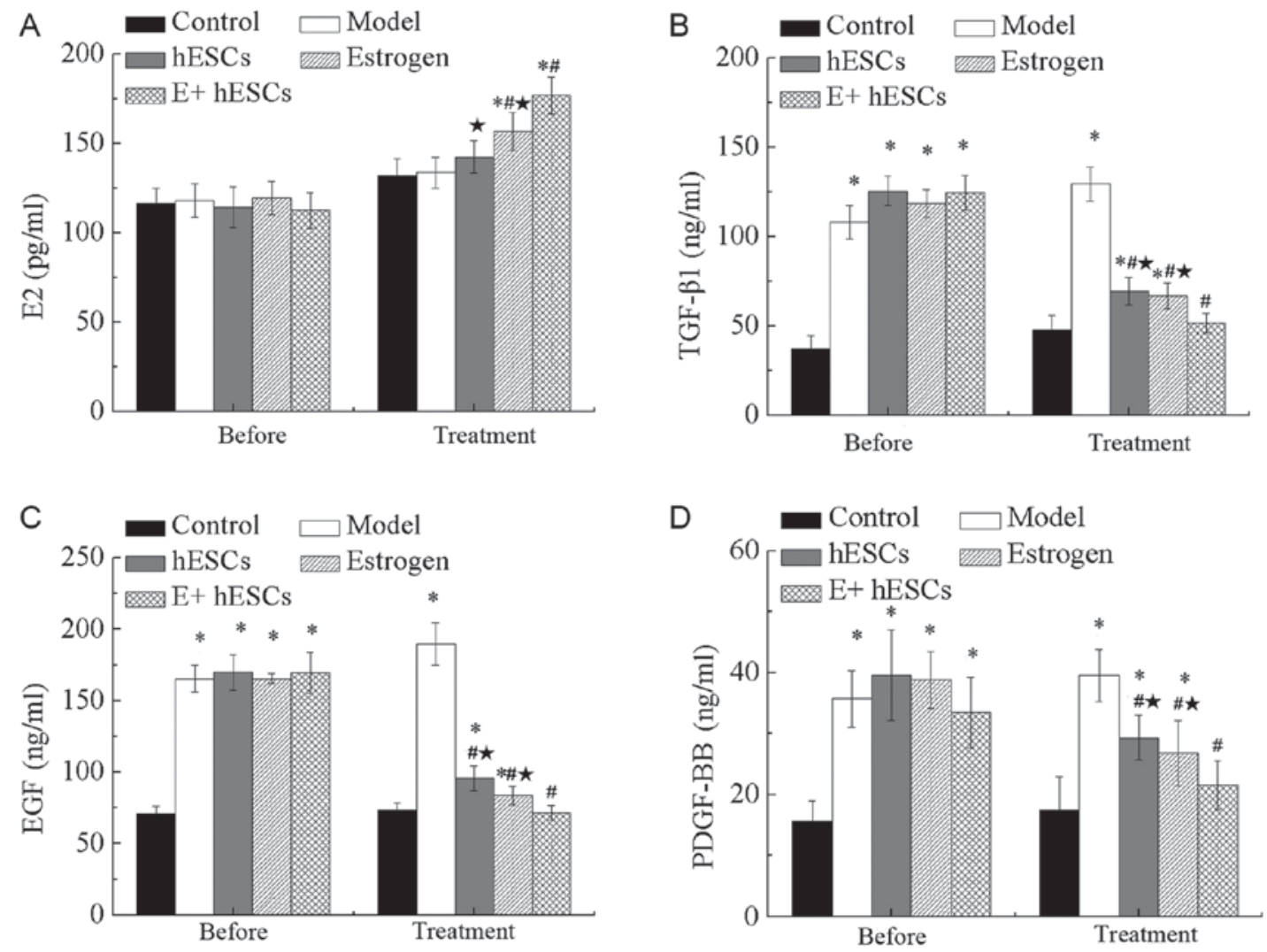

Figure 5. Serum levels of E2, TGF- $\beta 1$, EGF and PDGF-BB in a rat model of intrauterine adhesions. Expression of (A) E2, (B) TGF- $\beta 1$, (C) EGF and (D) PDGF-BB in the serum of rats. ${ }^{*} \mathrm{P}<0.05$ vs. control; ${ }^{*} \mathrm{P}<0.05$ vs. model; ${ }^{\star} \mathrm{P}<0.05$ vs. E+EnSCs. E+EnSCs, estrogen plus endometrial stem cells; EGF, epidermal growth factor; PDGF-BB, platelet-derived growth factor-BB; TGF- $\beta 1$, transforming growth factor- $\beta 1$; E2, $\beta$-estradiol. Before, 1 week after IUA; Treatment, 5 weeks after IUA.

\section{Discussion}

PDGF-BB has important functions in endometrial tissue remodeling (19). As a member of the EGF family, EGF determines cell survival, proliferation, differentiation and migration through interacting with the EGF receptor. EGF has also been found to be important for organ repair and wound healing (20). In the present study, expression levels of EGF and PDGF-BB in IUA tissues were significantly increased compared with those in the controls. Additionally, serum levels of EGF and PDGF-BB in rats with IUA were significantly increased compared with those of the controls. These results suggest that EGF and PDGF-BB may be involved in the development of IUA. Upregulation of TGF- $\beta$ is associated with the development of fibrotic diseases (21), and endogenous TGF- $\beta$ is essential for hypertrophic remodeling and the pathogenesis 
A
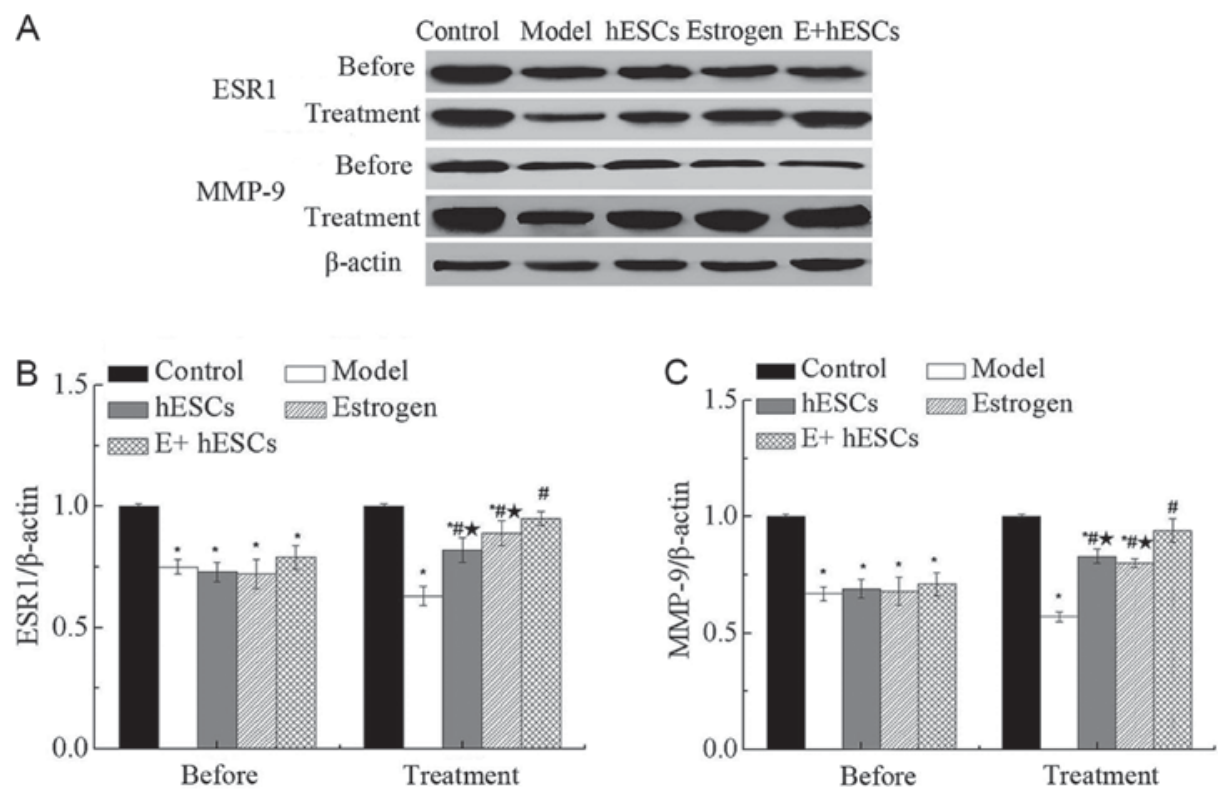

Figure 6. Protein expression of ESR1 and MMP-9 in a rat model of intrauterine adhesions. (A) Western blot analysis of ESR1 and MMP-9 expression in the endometrium of rats. (B) Relative expression level of ESR1. (C) Relative expression level of MMP-9. " $\mathrm{P}<0.05$ vs. control group; ${ }^{*} \mathrm{P}<0.05$ vs. model; ${ }^{\star} \mathrm{P}<0.05$ vs. E+EnSCs. E+EnSCs, estrogen plus endometrial stem cells; ESR1, estrogen receptor; MMP-9, matrix metalloproteinase-9. Before, 1 week after IUA. Treatment, 5 weeks after IUA.

of cardiac fibrotic remodeling (22). In the present study, it was identified that serum TGF- $\beta$ level significantly increased in rats with IUA compared with that in the sham surgery group. These results suggest that PDGF-BB, EGF and TGF- $\beta$ may be involved in the development of IUA.

Estrogen is able to increase the number of cells during endometrial injury and promote the regeneration of the uterine endometrium (23-25). In the present study, the level of E2 was significantly increased in the serum of rats with IUA compared with that in the controls, suggesting an effect of estrogen in the repair of intrauterine adhesions. Estrogen has been widely used in the prevention of IUA following operative hysteroscopy. Roy et al (26) reported that estrogen treatment reduced the incidence rate of IUA from 6.9 to $0 \%$. In the present study, estrogen treatment significantly prevented the IUA-mediated increase in the serum levels of TGF- $\beta 1$, EGF and PDGF-BB, indicating that estrogen may inhibit the development of IUA. Additionally, the serum levels of ESR 1 were significantly decreased in rats with IUA, whereas the level of ESR1 was significantly increased following estrogen treatment, also indicating that estrogen may inhibit the development of IUA. MMP-9 serves an important function in the remodeling of the endometrium (27). In the present study, the expression level of MMP-9 was significantly decreased in the rats with IUA compared with that in the controls. Additionally, morphological changes induced by IUA were partially reversed by estrogen treatment. These results suggest that estrogen may regulate the development of IUA.

Several studies (28-30) have reported that EnSCs may contribute to endometrial repair. Low proliferation and differentiation rate of EnSCs may lead to a variety of endometrial diseases, including endometriosis, endometrial polyps or even endometrial cancer (31). In the present study, EnSCs were isolated from ovulating women without endometrial disease, and the results demonstrated that the expression levels of endometrial epithelial stem cell-associated genes, including EMA, CK and CD49f, and endometrial stromal cell-associated genes, including THY-1, Col I, 5B5 and vimentin, were significantly increased in EnSCs compared with those in the controls. Additionally, hESC transplantation partially reversed the biochemical and morphological changes induced by IUA in vivo. These results indicate the clinical value of EnSCs transplantation in the treatment of IUA. Additionally, rats with IUA received EnSCs transplantation and estrogen treatment. The results demonstrated that EnSCs transplantation combined with estrogen significantly increased the treatment efficacy of IUA. These results indicate that EnSCs transplantation combined with estrogen treatment may achieve improved treatment outcomes compared with hESC transplantation alone.

Overall, the expression levels of EGF and PDGF-BB were significantly increased in the endometrium of patients with IUA compared with those in controls. EnSCs transplantation combined with estrogen improved the efficacy of treatment in vivo. The underling molecular mechanism may be associated with the decreased expression levels of EGF and PDGF-BB, and the increased expression levels of ESR1 and MMP-9.

\section{Acknowledgements}

Not applicable.

\section{Funding}

The present study was supported by the National Natural Science Foundation of China (grant no. 81601276), the Shandong Natural Science Foundation of China (grant no. ZR2016HL09) and the Shandong Medical and Health Science and Technology Development Project (grant no. 2015WS0033). 


\section{Availability of data and materials}

All data generated or analyzed during this study are included in this published article.

\section{Authors' contributions}

$\mathrm{XW}, \mathrm{HB}$ and $\mathrm{CH}$ designed the study. XW, HB, XL, CW and $\mathrm{CH}$ analyzed and interpreted the data. XW and $\mathrm{HB}$ wrote and revised the manuscript. All authors read and approved the final manuscript.

\section{Ethics approval and consent to participate}

All participants provided written informed consent and the study was approved by the Ethics Committee of The Affiliated Yantai Yuhuangding Hospital of Qingdao University.

\section{Consent for publication}

Not applicable.

\section{Competing interests}

The authors declare that they have no competing interests.

\section{References}

1. Deans R and Abbott J: Review of intrauterine adhesions. J Minim Invasive Gynecol 17: 555-569, 2010.

2. Schenker JG: Etiology of and therapeutic approach to synechia uteri. Eur J Obstet Gynecol Reprod Biol 65: 109-113, 1996.

3. Salma U, Xue M, Ali Sheikh MS, Guan X, Xu B, Zhang A, Huang $\mathrm{L}$ and $\mathrm{Xu} \mathrm{D}$ : Role of transforming growth factor- $\beta 1$ and smads signaling pathway in intrauterine adhesion. Mediators Inflamm 2016: 4158287, 2016.

4. Chen F, Duan H, Zhang Y and Wu YH: Effect and mechanism of formation of intrauterine adhesion at different dose of estrogen. Zhonghua Fu Chan Ke Za Zhi 45: 917-920, 2010 (In Chinese).

5. Di Spiezio Sardo A, Mazzon I, Bramante S, Bettocchi S, Bifulco G, Guida M and Nappi C: Hysteroscopic myomectomy: A comprehensive review of surgical techniques. Hum Reprod Update 14: 101-119, 2008.

6. Johary J, Xue M, Zhu X, Xu D and Velu PP: Efficacy of estrogen therapy in patients with intrauterine adhesions: Systematic review. J Minim Invasive Gynecol 21: 44-54, 2014.

7. Capella-Allouc S, Morsad F, Rongieres-Bertrand C, Taylor S and Fernandez H: Hysteroscopic treatment of severe Asherman's syndrome and subsequent fertility. Hum Reprod 14: 1230-1233, 1999.

8. Ding L, Li X, Sun H, Su J, Lin N, Péault B, Song T, Yang J, Dai J and $\mathrm{Hu}$ Y: Transplantation of bone marrow mesenchymal stem cells on collagen scaffolds for the functional regeneration of injured rat uterus. Biomaterials 35: 4888-4900, 2014.

9. Jing Z, Qiong Z, Yonggang W and Yanping L: Rat bone marrow mesenchymal stem cells improve regeneration of thin endometrium in rat. Fertil Steril 101: 587-594, 2014.

10. Zhou BO, Yue R, Murphy MM, Peyer JG and Morrison SJ: Leptin-receptor-expressing mesenchymal stromal cells represent the main source of bone formed by adult bone marrow. Cell Stem Cell 15: 154-168, 2014.

11. Zhang Y, Lin X, Dai Y, Hu X, Zhu H, Jiang Y and Zhang S: Endometrial stem cells repair injured endometrium and induce angiogenesis via AKT and ERK pathways. Reproduction 152: 389-402, 2016
12. Yun BH, Jeon YE, Chon SJ, Park JH, Seo SK, Cho S, Choi YS, Lee JS and Lee BS: The prognostic value of individual adhesion scores from the revised American fertility society classification system for recurrent endometriosis. Yonsei Med J 56: 1079-1086, 2015.

13. Livak KJ and Schmittgen TD: Analysis of relative gene expression data using real-time quantitative PCR and the 2(-Delta Delta C(T)) method. Methods 25: 402-408, 2001.

14. Ebrahimi-Barough S, Massumi M, Mohseni Kouchesfahani $\mathrm{H}$ and Ai J: Derivation of pre-oligodendrocytes from human endometrial stromal cells by using overexpression of MicroRNA 338. J Mol Neurosci 51: 337-343, 2013.

15. Ebrahimi-Barough S, Mohseni Kouchesfahani H, Ai J, Mahmoodiniac M, Tavakole S and Massumic M: Programming of human endometrial-derived stromal cells (EnSCs) into pre-oligodendrocyte cells by overexpression of miR-219. Neurosci Lett 537: 65-70, 2013.

16. Tang YQ, Gan L, Xu Q, Wang S, Li JJ and Duan H: Effects of human umbilical cord mesenchymal stem cells on intrauterine adhesions in a rat model. Int J Clin Exp Pathol 9: 12119-12129, 2016.

17. You Z, Sun J, Xie F, Chen Z, Zhang S, Chen H, Liu F, Li L, Chen G, Song Y, et al: Modulatory effect of fermented papaya extracts on mammary gland hyperplasia induced by estrogen and progestin in female rats. Oxid Med Cell Longev 2017: 8235069, 2017.

18. Zhou Y, Kim YS, Chakraborty S, Shi J, Gao H and Liu S: 99mTc-labeled cyclic RGD peptides for noninvasive monitoring of tumor integrin $\alpha_{\mathrm{v}} \beta_{3}$ expression. Mol Imaging 10: 386-397, 2011.

19. Matsumoto H, Nasu K, Nishida M, Ito H, Bing S and Miyakawa I: Regulation of proliferation, motility, and contractility of human endometrial stromal cells by platelet-derived growth factor. J Clin Endocrinol Metab 90: 3560-3567, 2005.

20. Martin P and Nunan R: Cellular and molecular mechanisms of repair in acute and chronic wound healing. Br J Dermatol 173: 370-378, 2015

21. Qin W, Chung AC, Huang XR, Meng XM, Hui DS, Yu CM, Sung JJ and Lan HY: TGF- $\beta /$ Smad3 signaling promotes renal fibrosis by inhibiting miR-29. J Am Soc Nephrol 22: 1462-1474, 2011.

22. Mascareno E, Galatioto J, Rozenberg I, Salciccioli L, Kamran H, Lazar JM, Liu F, Pedrazzini T and Siddiqui MA: Cardiac lineage protein-1 (CLP-1) regulates cardiac remodeling via transcriptional modulation of diverse hypertrophic and fibrotic responses and angiotensin II-transforming growth factor $\beta$ (TGF- $\beta 1$ ) signaling axis. J Biol Chem 287: 13084-13093, 2012.

23. Hyodo S, Matsubara K, Kameda K and Matsubara Y: Endometrial injury increases side population cells in the uterine endometrium: A decisive role of estrogen. Tohoku J Exp Med 224: 47-55, 2011.

24. Wang Z, Cui Y, Chen Y, Zhang D, Liang Y, Zhang D, Wu Q, Xie J, Ouyang S, Li D, et al: Comparative genetic mapping and genomic region collinearity analysis of the powdery mildew resistance gene Pm41. Theor Appl Genet 127: 1741-1751, 2014.

25. Jecker NS: Response to open peer commentaries on 'Justice between age groups: An objection to the prudential lifespan approach'. Am J Bioeth 14: W10-W12, 2014.

26. Roy KK, Negi N, Subbaiah M, Kumar S, Sharma JB and Singh N: Effectiveness of estrogen in the prevention of intrauterine adhesions after hysteroscopic septal resection: A prospective, randomized study. J Obstet Gynaecol Res 40: 1085-1088, 2014

27. Skinner JL, Riley SC, Gebbie AE, Glasier AF and Critchley HO: Regulation of matrix metalloproteinase-9 in endometrium during the menstrual cycle and following administration of intrauterine levonorgestrel. Hum Reprod 14: 793-799, 1999.

28. Du H and Taylor HS: Stem cells and female reproduction. Reprod Sci 16: 126-139, 2009.

29. Gargett CE and Ye L: Endometrial reconstruction from stem cells. Fertil Steril 98: 11-20, 2012.

30. Gargett CE, Nguyen HP and Ye L: Endometrial regeneration and endometrial stem/progenitor cells. Rev Endocr Metab Disord 13: 235-251, 2012.

31. Maruyama T and Yoshimura Y: Stem cell theory for the pathogenesis of endometriosis. Front Biosci (Elite Ed) 4: 2754-2763, 2012. 